\title{
Evaluation of the Transfer of Heat From the Coil of the LHC Dipole Magnet to Helium II
}

\author{
David Richter, Jerôme Fleiter, Bertrand Baudouy, and Arnaud Devred
}

\begin{abstract}
During operation of the Large Hadron Collider at CERN, heat will be generated inside the coils of its superconducting magnets as a consequence of ramping of magnetic field, and of the interaction of lost beam particles with the magnet mass. Heat has to be transferred from the conductor into the He II coolant and removed from the magnet environment.

During the LHC R\&D stage, this transfer has been extensively studied on simulated coil segments at CEA/Saclay, and by analyzing dynamic behavior of short model magnets at CERN.

Owing to the importance of efficient cooling for the design of future superconducting accelerator magnets, study of heat transfer has been restored at CERN and in frame of the Next European Dipole Collaboration.

The article features two recently performed works:

1) Attempt to analyse archived high ramp rate quench data of 1-m-long LHC model dipole magnets of the 2 nd generation.

2) Development of a method for direct measurement of heat transfer on segments of production LHC dipole magnet coils.
\end{abstract}

Index Terms-Cooling, He II, high ramp rate quenching, superconducting magnets.

\section{INTRODUCTION}

$\mathbf{T}$ HE Large Hadron Collider (LHC), presently under construction at CERN, Geneva, comprises 1232 main superconducting (SC) dipoles and 392 main SC quadrupoles [1].

Graded coils, Fig. 1, of the SC dipoles consist of two layers wound with an Nb-Ti based, state of the art Rutherford-type SC cable. Within a pole, the cable is organized in 6 blocks. The inner layer cable counts 28 strands, the outer layer one 36 .

The cable is insulated with two layers of polyimide insulation (Fig. 2). The internal layer is made of two $50 \%$ overlapped, $50-\mu \mathrm{m}$-thick, $11-\mathrm{mm}$-wide tapes. The outer layer is a single, $69-\mu \mathrm{m}$-thick, 9 -mm-wide polyimide tape coated by polyimide glue on its external surface. It is wound leaving a $2-\mathrm{mm}$-wide gap between turns in order to facilitate penetration of $\mathrm{He}$ II inside the coil.

Manuscript received August 29, 2006. The tests reported were performed at CERN. This work was supported in part by the European Community-Research Infrastructure Activity under the FP6 "Structuring the European Research Area" program (CARE, contract number RII3-CT-2003-506395).

D. Richter is with the CERN, AT Division, 1211 Geneva 23, Switzerland (e-mail: david.richter@cern.ch).

J. Fleiter, on a training course at CERN during the reported work, is with Ecole Normale Supérieure, master Physique, Théorie Expérience et Modèle, 94235 Cachan, France.

B. Baudouy is with CEA-Saclay, DSM/DAPNIA/SACM, 91191 Gif-sur-Yvette Cedex, France.

A. Devred is with CEA-Saclay, DSM/DAPNIA/SACM, 91191 Gif-surYvette Cedex, France, and also with CERN, AT Division, 1211 Geneva 23, Switzerland.

Digital Object Identifier 10.1109/TASC.2007.898030

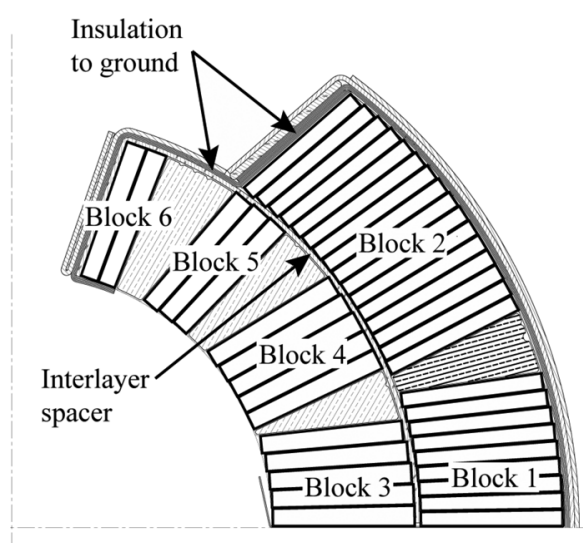

Fig. 1. Quadrant of the LHC main dipole coil (six block geometry).

The polyimide insulation to ground and the grooved GFRP interlayer spacer, Fig. 1, complete the coil insulation scheme.

During operation, heat will be generated inside the SC coils operating at $1.9 \mathrm{~K}$ as a consequence of ramping of magnetic field, and of the interaction of lost beam particles with the magnet mass. All heat has to be transferred from the conductor towards the He II coolant and removed from the magnet environment. The cable insulation determines the efficiency of heat transfer (HT) from the cable towards the He II.

Development of the LHC main dipole magnet relied on large number of 1-m-long model magnets. Models built by industry before 1994 are referred to as the models of the 1st generation [2], models built by CERN after 1994 are referred to as the models of the 2nd generation [3].

In the LHC R\&D stage, HT has been studied on simulated coil segments by CEA/Saclay (CEA) [4]-[8], and by analyzing dynamic behavior of 1-m-long model magnets of the 1st generation at CERN [9].

Owing to the importance of heat removal in SC magnets, study of heat transfer following both methods has been restored at CERN, and in the frame of the Next European Dipole Collaboration (NED). The goal is to fully exploit data available for the LHC magnets under completion, and to gather information required for the design of LHC upgrade magnets.

In the article, we report

- On new results on HT obtained by analyzing the high ramp rate quench current sensitivity of the 2nd generation, $1-\mathrm{m}$ long LHC model magnets of the 5-block design, and

- on the first results obtained by a direct HT measurement method on a segment of production LHC dipole coil. 


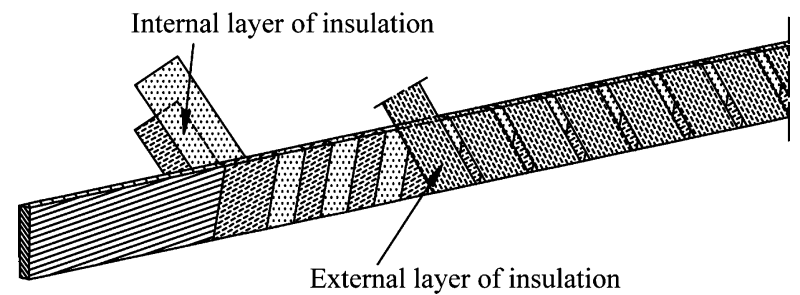

Fig. 2. The internal and external layers of insulation of the SC cable in the LHC main dipoles.

When comparing with the results previously obtained by CEA, we rely on the sample denomination used in ref. [4]-[8], e.g. 'A15', 'A25', 'A30', 'A32', and 'A34bis'.

\section{Heat Transfer Measurement Performed During the LHC R\&D PERIOD AND AT PRESENT}

When in stationary conditions heat power, $P_{\text {heat }}$, is generated in a magnet conductor immersed in a cooling bath at the temperature, $T_{\text {bath }}$, the conductor temperature attains a value $T_{\text {cond }}>T_{\text {bath }}$. Then, efficiency of the transfer of heat from the conductor to the cooling bath is described by the temperature difference $\Delta T_{\mathrm{HT}}=T_{\text {cond }}-T_{\text {bath }}$ as function of the heating power, $P_{\text {heat }}$, with $T_{\text {bath }}$ as a parameter.

In the case of a SC magnet coil, measurement of stationary HT in situ, i.e. acquisition of the $\Delta T_{\mathrm{HT}}\left(P_{\text {heat }}\right)$ curve, would give invaluable results but is nearly impossible to perform. The need to determine with confidence the heating power and the temperature within a heterogeneous coil section imposes restrictions on the configuration of the experiment. Suitable techniques, approaching real situation, have been developed.

\section{A. Direct Method on Simulated Coil}

In the direct HT measurement performed at CEA [4], stainless steel plates were machined into the shape of SC cable, instrumented with temperature sensors and wrapped with insulation. The ends of the plates were not machined in order to ensure tightness of the insulating tapes and thus prevent $\mathrm{He}$ from forming cooling channels at the extremities. The magnet coil was represented by a stack of five such plates under appropriate pressure, immersed into a He II bath. Temperatures in the plates and in the bath were measured as a function of the heating power generated by passing DC electrical current through the plates.

An extensive comparative study of pre-selected insulating schemes was done between 1990 and 2000 [4]-[8].

\section{B. Stationary Analysis of Magnet Quenching}

At CERN, conclusions about the transfer of heat from the coil to He II were drawn from analysis of dynamic behavior of the 1st generation LHC 1-m-long model magnets [9].

When current is slowly ramped up in a cooled SC magnet, its internal temperature remains almost constant. Magnetic field increases and the current carrying capacity of the conductor decreases until finally, a quench appears at the region of the highest field.

If the magnet current is ramped up fast [10], i.e. several hundreds of $\mathrm{A} / \mathrm{s}$ in our case, the conductor heats up due to the electromagnetic power loss. The coil becomes warmer than the coolant and the temperature profile deforms. Both temperature and magnetic field variations affect the current carrying capacity and determine the location where the quench originates. The higher the ramp rate, the lower the quench current. High Ramp Rate Quench (HRRQ) sensitivity is an important magnet characteristic, and was thoroughly studied.

Heating of magnet coils in ramping magnetic field was investigated at first. Main sources were the inter-strand coupling loss, and, to a lesser extent, the intra-filament hysteretic loss. Electromagnetic losses were regularly measured on the R\&D magnets using the electrical integration method [9].

If the location of the quench origin within a coil is known in the HRRQ experiment, the local magnetic field can be deduced from the magnetic field map and from the quench current. Using the field time derivative, heating power can be deduced from the magnet electromagnetic loss data. Finally, temperature at the quench origin can be deduced from the critical surface of the magnet conductor. Temperature of the bath can be reliably measured owing to the thermal homogeneity of He II. In that way, the HT data for a magnet coil can be deduced, each quench giving one point on the $\Delta T_{\mathrm{HT}}\left(P_{\text {heat }}\right)$ curve.

Results for two, 1st generation, 1-m-dipole model magnets were published in [9].

\section{Direct Method on Coil Segment}

Our present method is a combination of the above two.

The sample is a segment from a production coil of an LHC main dipole. Temperature of the superconductor is measured using arc-welded $\mathrm{AuFe}_{0.07 a t \%} /$ Chromel thermocouples. Heat is generated in a way inspired by the DC method used for the interstrand contact resistance measurement [11]. Current is injected into a few strands of the Rutherford-type cable, generating heat in the contacts between all strands.

\section{EVAluation of HEAT TRANSFER From HRRQ Data}

\section{A. Data Reduction}

About 34 1-m-long model dipole magnets of the 2nd generation, denominated MBSMS1 to 23 and MBSMT1 to 11, were produced at CERN from 1995 to 1999, [3], [12]. They have been assembled with one, or with two apertures, in the five block, and in the six block coil geometries (Fig. 1). They relied on different conductors, mechanical structures, assembly procedures, and instrumentation. Several versions of the same model were made using the same set of coils.

The models were extensively tested in vertical Claudet-bath cryostats, [3], [12]-[14]. Without the beam pipe installed, He II freely accessed the inner surface of the coils, which is the main cooling surface in LHC dipole magnets. A huge amount of data has been collected. Our task was to find data relevant for the evaluation of the HT and analyse them in the same way as done for the models of the 1st generation [9].

The summary quench data are available in Excel files of standardized format, 1 file per magnet and per version. Electromagnetic losses were measured on one version of each early model. 
Also available are conductor critical current data and comprehensive design and test documentation.

In order to allow comparison across all the different species, the quench files have been loaded into an Oracle database. All single aperture magnet data have been treated (6114 quench records).

Results of the electromagnetic loss measurements and of the conductor critical current measurements have been loaded into dedicated tables, as have been maps of magnetic field.

The Oracle database was used for most calculations:

- Local magnetic field components, both $\|$ and $\perp$ to the cable face, and their time derivative were calculated from the field maps.

- As measured, the coupling loss is an integral value over the whole coil. In HRRQ analysis, the measured value was first adjusted to the actual current ramp rate. Then, the loss determined for the whole coil was scaled down to the turn where the quench originated, using a map of coupling loss derived from the magnetic field map.

- The hysteretic loss was calculated for every strand from the strand geometry and from the local strand critical current, and then summed over the turn of quench origin.

- The conductor critical surface was generated from critical current values measured at $1.9 \mathrm{~K}$ and at $4.222 \mathrm{~K}$ according to the parameterization of [15].

\section{B. Evaluation of the HT Characteristics}

From all performed HRRQ, only those fulfilling all of the following conditions were used for the HT analysis:

- HRRQ on magnets that terminated their training in order that the quench current is a unique function of $T_{\text {cond }}$ and $B_{\text {cond }}$, where $B_{\text {cond }}$ is the magnetic field at the conductor.

- HRRQ resulting from a ramp with pre-cycle as shown in Fig. 3, in order that all strands in a cable carry the same current, which has not been altered by the Boundary Induced Coupling Currents (BICCs) [9].

- HRRQ for which the turn and the longitudinal position of their origin were indicated by the test operator. The turn may be deduced from a block number.

- HRRQ that originated in the straight part of the coil, far from the joint between the inner and outer coil.

- HRRQ on magnets, for which the electromagnetic loss was measured.

Among the single aperture models, the MBSMS4, 5, 6, 7, and 8 , version 1 magnets met all criteria. Remaining quenches in the joint area were discarded.

Fig. 4 shows the HRR quenches of the MBSMS4, 5, 6, 7, and 8 version 1 model magnets at $T_{\text {bath }}=1.9 \mathrm{~K}$. Fig. 5 shows the HT curves resulting from their analysis. The originally distant curves get closer. The transformation from the 'Current ramp rate' abscissa to the 'Total loss' abscissa removes difference in inter-strand contact resistance, which varies between $3.0 \mu \Omega$ and $6.5 \mu \Omega[16]$ among the compared magnet coils.

The origin of the somewhat higher $\Delta T_{\mathrm{HT}}$ in MBSMS5 is not clear. All compared magnets have similar overall specific coupling loss rate in the range of $(0.29-0.62) \times 10^{-3} \mathrm{~J}$. $\mathrm{s} / \mathrm{m} / \mathrm{A}^{2}[16]$, in the measurement units [9], with the largest loss

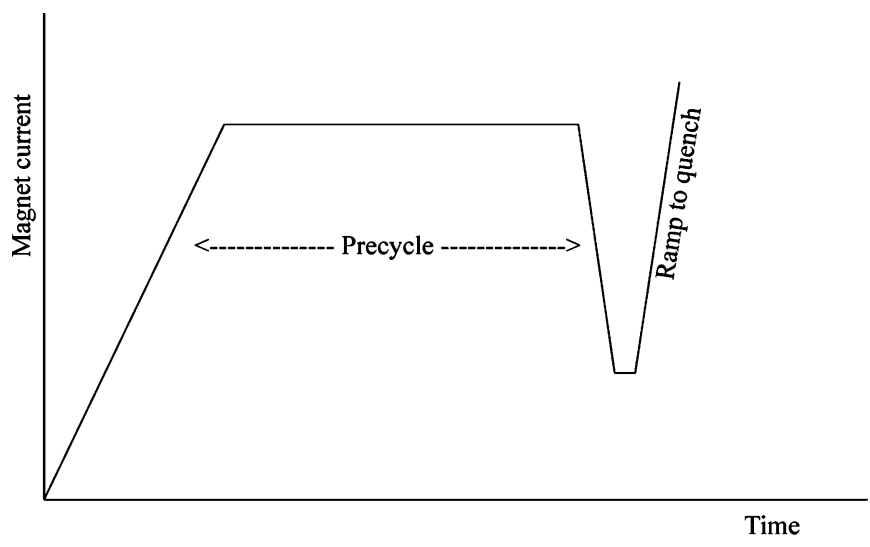

Fig. 3. The magnet current ramp with pre-cycle [9].

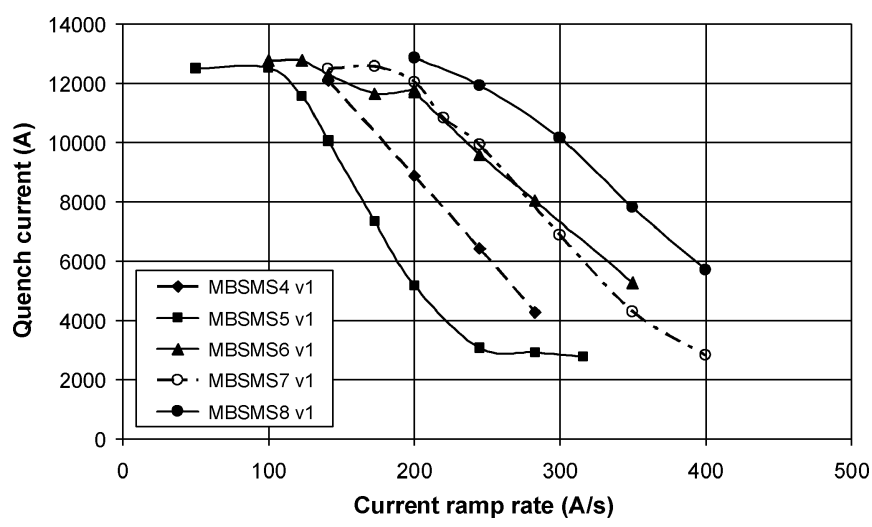

Fig. 4. HRRQ with pre-cycle quench data for the MBSMS4, 5, 6, 7, and 8 version 1 model magnets at $T_{\text {bath }}=1.9 \mathrm{~K}$.

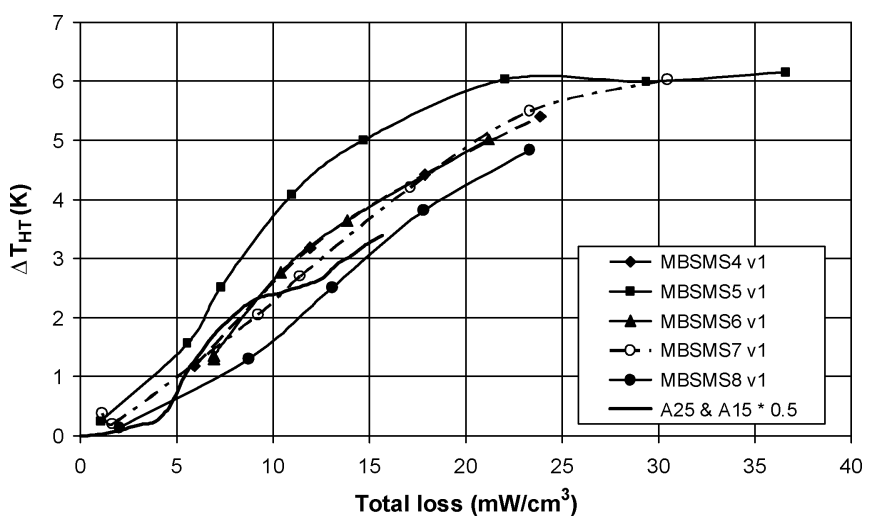

Fig. 5. HT data for the MBSMS4, 5, 6, 7, and 8 version 1 model magnets at $T_{\text {bath }}=1.9 \mathrm{~K}$. For comparison is shown the 'A $25 \& \mathrm{~A} 15 * 0.5$ ' HT curve. It was compiled from CEA data [4]-[8], with the heating power scaled by a factor of 0.5 .

in MBSMS5. In the group under evaluation, the MBSMS5 version 1 magnet attained the lowest training quench currents and MBSMS8 version 1 magnet the highest ones, with the difference between them of up to $600 \mathrm{~A}$.

For MBSMS4, 6, and 8, the production cable critical data were used. For MBSMS5 and 7, the cable critical data have been re-measured for the analysis. Agreement between predicted short sample limit and the maximum $1.9 \mathrm{~K}$ magnet quench current is better than $1.6 \%$ with the exception of 
MBSMS5, where the maximum quench current is $4.6 \%$ below the predicted value.

The MBSMS5 behavior is not a mathematical consequence of any cable critical current problem, as scaling of the cable critical data to the maximum training quench current results in a reduction of the $T_{\text {cond }}$ temperature only by $0.07 \mathrm{~K}$. Note that MBSMS8 relies on a different polyimide insulation supplier.

Unlike insulation in the LHC main dipoles, the internal layer of insulation (Fig. 2) in the inner coils of the MBSMS4, 5, 6, 7 , and 8 magnets was made of two $48 \%$ overlapped, $25-\mu \mathrm{m}-$ thick, $15-\mathrm{mm}$-wide polyimide tapes. The HT curve for similar insulation measured by CEA [4]-[8] has been added in Fig. 5 . It was compiled from the data for the A25 sample for which the temperature difference, $\Delta T_{\mathrm{HT}}<0.25 \mathrm{~K}$. For $\Delta T_{\mathrm{HT}}>0.25 \mathrm{~K}$, the curve has been extended using the data for the A15 insulation, having similar performance in the first area. The transferred power of the curve data was multiplied by a factor of 0.5 to obtain agreement with the MBSMS4, 5, 6, 7, and 8 data. The curve is based on a large number of measurements. The changes of slope reflect the change of the HT regime when the sample reaches the temperature $T_{\lambda}$, or when it reaches the temperature of boiling He I.

The MBSMS4, 5, 6, 7, and 8 HT data (Fig. 5), rely on HRRQ originating in Block 3 (Fig. 1), near the inner coil mid-plane. The inner surface of the inner coil is exposed to the He II bath. Through grooves in the interlayer spacer (Fig. 1), He II can also wet the outer surface of the inner coil. However, all analysed HRRQ originated at, or above $T_{\lambda}$, at the most unfavorable location with respect to the interlayer spacer. This could impair the cooling on the outer surface of Block 3, thus explaining the factor 0.5 , introduced to achieve agreement between HRRQ and CEA data.

Agreement of the HT characteristics deduced from the HRRQ sensitivity with the CEA measurement seems reasonable, in particular if we take into account the difference between the two measurement methods, and the sensitivity of the HT properties to the preparation of the coils and the test samples. The lowest $\Delta T_{\mathrm{HT}}$ data are difficult to obtain in HRRQ test and probably less reliable. They rely on quench currents approaching the training quench limit, which causes magnets to quench for reasons other than heating by electromagnetic loss.

The error associated with this method was estimated to be less than $0.5 \mathrm{~K}$ [9]. Possible sources of uncertainty are the BICCs, variation of strands within the early production cable, conductor data measured at $1.9 \mathrm{~K}$ and $4.222 \mathrm{~K}$ and scaled to much higher temperatures, non-homogeneity of the power loss over the coil, etc.

\section{Measurement Of HT On a Segment of an LHC DIPOLE COIL}

\section{A. Sample Preparation}

The test sample was a $300 \mathrm{~mm}$ long segment of the Block 3 , shown in Fig. 1, of a production LHC main dipole coil manufactured by BNN, Germany. It consists of 5 insulated inner layer cables joined together by polyimide glue.

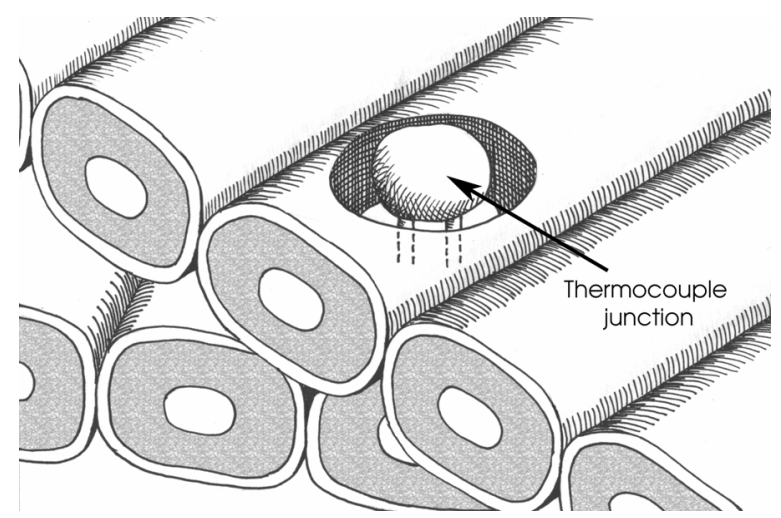

Fig. 6. Detail of a TCJ mounted inside a SC strand.

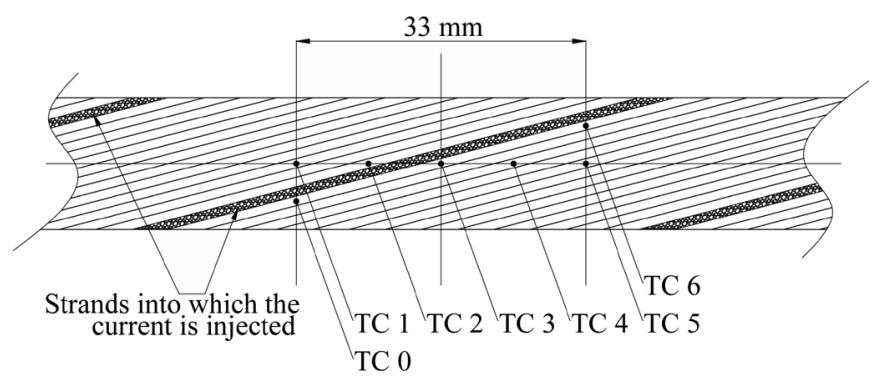

Fig. 7. Position of the TCJs in the central area of the central cable.

The central, i.e. 3rd cable was instrumented with an array of 7 thermocouple junctions (TCJs), installed on the central part of the conductor as seen in Figs. 6 and 7.

To do so, the external layer (Fig. 2) of the insulation of the 4 th cable in the stack was cut along the cable's wide edge. The 4th cable was temporarily taken out from the stack, while its internal layer of the insulation remained intact.

Seven $\varnothing 0.6 \mathrm{~mm}$ holes were drilled into selected strands of the 3rd cable (Fig. 6). Care was taken to avoid contamination of the environment by metallic splinters, or any deformation of the polyimide insulation.

The varnish insulated TCJs were installed in the holes, which, in turn, were sealed using PET thermal glue. For better tightness, the insulation of the cable was removed in the vicinity of the holes, so that the seal adhered to the metal of the conductor.

3-mm-thick fiberglass plates were added on each side of the stack, serving as a thermal separator and as a mechanical structure element. The sandwich was enclosed in a stainless steel holder under a pressure of $20 \mathrm{MPa}$ at room temperature. In order to restrict the access of liquid He, the stack and the fiberglass plates were sealed together at both extremities by the PET thermal glue on a length of about $7 \mathrm{~mm}$.

\section{B. Heating and Thermal Field}

Heat in the sample is generated by DC currents flowing through resistive inter-strand contacts. To inject the current, various electrical schemes can be envisaged. We used the one giving the most non-homogeneous power distribution but producing most power for a given current: Current was injected into the two most opposite strands of each 28 -strand cable sample. As the power is generated mainly in the crossover 


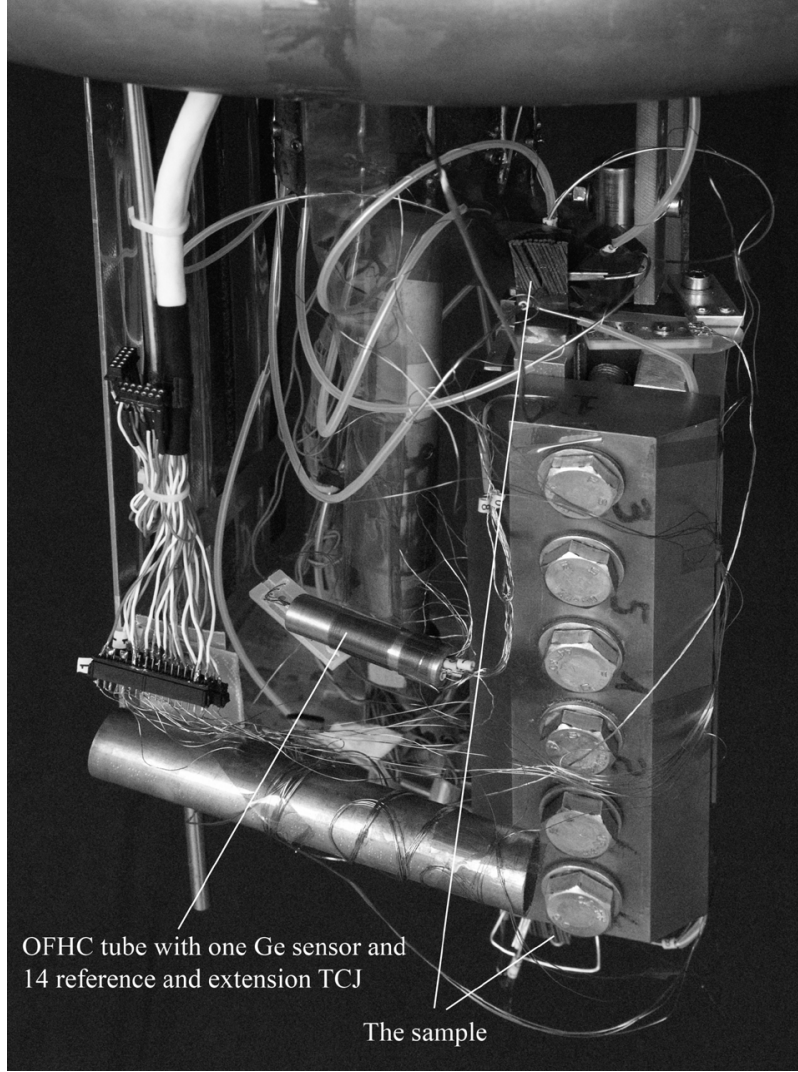

Fig. 8. The sample installed for testing.

contacts, the heating of the strands in which current is injected is 14 times that of the remaining strands.

To keep the evaluation of the HT data simple, it is desirable that the differences in temperature between strands of the heated cable be negligible. One of the goals of this experiment was to find out, if redistribution of heat inside the sample is sufficient to allow such approximation.

\section{Preparation of the Experiment}

The thermocouple wires were extended by copper lead wires [17]. All thermocouple-to-lead junctions were placed together inside an OFHC copper tube, instrumented with a Ge temperature sensor (Fig. 8). Another two Ge sensors measured the $\mathrm{He}$ temperature at both extremities of the sample.

The holder was installed in a Claudet-type cryostat [18]. Its $0.1 \mathrm{MPa}$ pressurized cold bath was regulated to temperature of $1.900 \pm 0.002 \mathrm{~K}$. Up to $1 \mathrm{kA}$ heating current was fed either into the central cable, or the three central cables, or all five cables through He-gas-cooled current leads.

Prior to measuring the described sample, we re-tested the A34bis sample, the last one in the series built by CEA [4]-[8]. In doing so we developed our skills and gained experience with the test facility.

\section{Results}

1) Estimation of Errors: The bath temperature, $T_{\text {bath }}$, was measured with an absolute precision of $10 \mathrm{mK}$. The $\Delta T_{\mathrm{HT}}$ difference was measured directly by the thermocouples. The parasitic thermal voltage introduced by the thermocouple lead wires

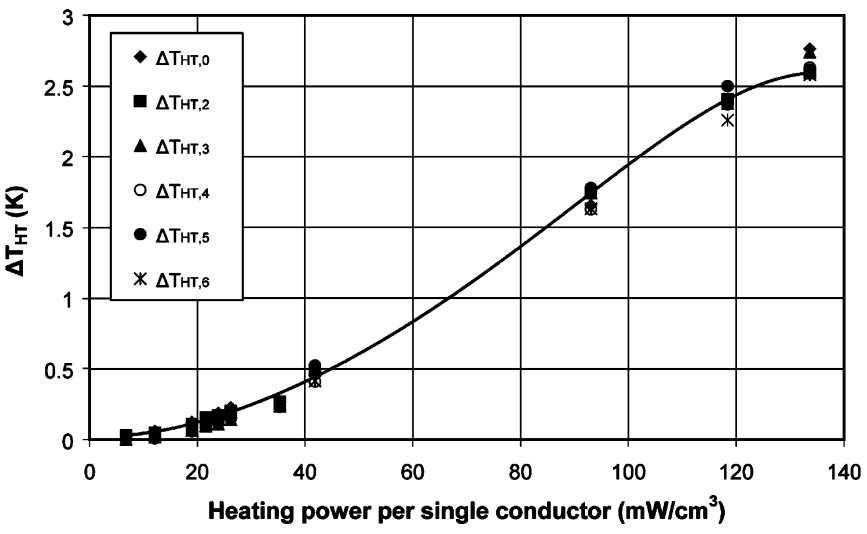

Fig. 9. HT data measured at $T_{\text {bath }}=1.90 \mathrm{~K}$, all five conductors heated. $\Delta T_{H T, 0}, \Delta T_{H T, 2}, \Delta T_{H T, 3}$, and $\Delta T_{H T, 6}$, were measured on strands next to the most heated strand, $\Delta T_{H T, 4}$, and $\Delta T_{H T, 5}$ in the colder pool.

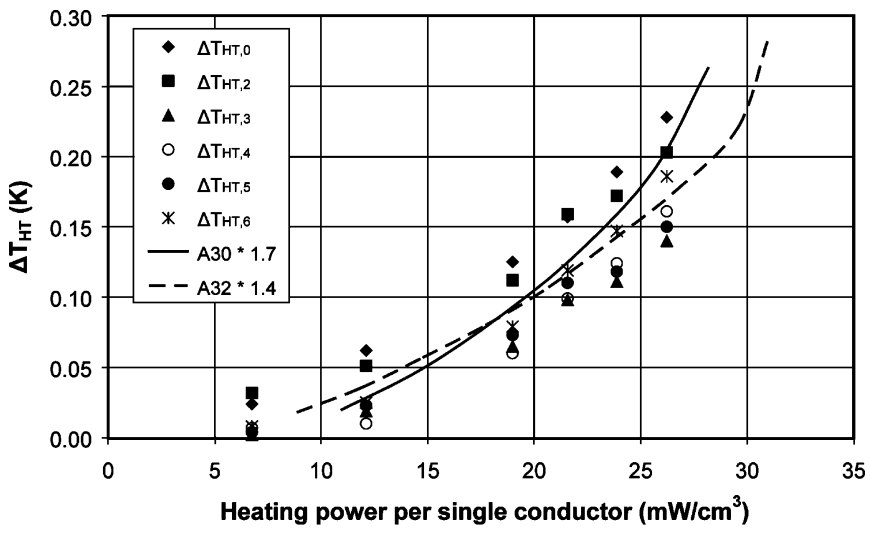

Fig. 10. Zoom of Fig. 9. For comparison are shown the 'A $30 * 1.7$ ' and 'A $32 *$ 1.4 ' curves measured at CEA [4]-[8]. In order to match our measurement, the heating power was scaled up 1.7 and 1.4 times respectively.

was tested to be less than $\pm 200 \mathrm{nV}$. Voltage drift of the thermocouple measurement was $200 \mathrm{nV}$, corresponding to $\pm 20 \div$ $30 \mathrm{mK}$ depending on the measured temperature. The $P_{\text {heat }}$ was calculated from the heating current and from voltage on the sample terminals with an overall error of less than $0.1 \%$. The principal source of error in our measurement was not the instruments but possible parasite heat leaks and electromagnetic noise on the TCJs.

2) Measurements: Figs. 9 and 10 show the $\Delta T_{\mathrm{HT}}\left(P_{\text {heat }}\right)$ curves measured when all five conductors are heated in a $1.90 \mathrm{~K}$ He II bath. Fig. 10 is a zoom of Fig. 9. The HT behavior depends on whether the sample temperature is below, or above $T_{\lambda}$.

3) Thermal Field: The difference between the temperature of a 'hot' strand and the rest of the cable is about $50 \mathrm{mK}$, as can be seen by comparing the $\Delta T_{\mathrm{HT}, 0}, \Delta T_{\mathrm{HT}, 2}, \Delta T_{\mathrm{HT}, 3}, \Delta T_{\mathrm{HT}, 6}$ data points, measured on the neighbors of the most heated strand, to the $\Delta T_{\mathrm{HT}, 4}, \Delta T_{\mathrm{HT}, 5}$, measured on the less heated strands. The cable compensates for most of the heating power non-uniformity.

The impact of heating 1 , or 3 , or all 5 cables resembles results obtained by CEA. 


\section{E. Discussion}

1) Sample Temperature Below $T_{\lambda}$ : Below $T_{\lambda}$, the HT curves follow closely those measured at CEA for the samples A30 and A32, providing the power data are re-scaled by a factor 1.7 and 1.4 , respectively. There is 1.4 to 1.7 times more power transferred from our sample than from the CEA one.

The origin of the difference may be partly traced to the preparation of our sample: lower transverse pressure than in the CEA sample, longer sample active length than expected due to the influence of the extremities, excessive contact of the thermocouples with coolant, or some extra access for He II into the sample, created by excessive manipulation during instrumentation. Part of the effect, however, might have a physical meaning. The interior of a real cable is composed of a network of inter-connected cavities filled with He II. If there were an efficient cooling path from the cable interior to the He II bath, a real coil would have had a heat exchange surface larger than in the simulated coil CEA experiment.

2) Sample Temperature Crossing $T_{\lambda}$ and Above: In the CEA experiment, crossing $T_{\lambda}$ is marked by a sharp increase in slope of the $\Delta T_{\mathrm{HT}}\left(P_{\text {heat }}\right)$ curves. In our test, the change is mild and the crossing is fuzzy. Above $T_{\lambda}$, our curves correspond to 5 to 15 time higher transferred power than in the CEA experiments.

On each side of the active part of the sample, outside the 7 $\mathrm{mm}$ PET seal, $4 \mathrm{~cm}$ of cable have been left for electrical connections. A barrier against He was foreseen but not installed during the sample preparation. Heat conducted along strands towards the well cooled extremities might cause the unexpected result above $T_{\lambda}$. Below $T_{\lambda}$, this conduction has relatively much less importance, and the effect is attenuated.

\section{F. Effect of Sample Preparation and Testing}

Based on the preliminary analysis of the results, the following measures should be considered:

- Reduction of the electrical noise of the TCJ voltage acquisition;

- Closing any possible parasitic cooling channels;

- Manipulating the sample less during preparation, in order not to alter its properties;

- Use of powering scheme offering less difference between the most powered strands and the colder pool.

\section{CONCLUSIONS}

We have analysed the heat transfer properties of a number of $1 \mathrm{~m}$ long LHC model dipoles and devised a method for measurement of HT on segments of magnet coils.

Both methods gave results that can be understood on the basis of the previous measurements at CEA, [4]-[8].

Evaluation of HT from HRRQ data is a unique method rendering HT data of a real magnet in close-to-real heating conditions. According to the condition of the magnet, it may be difficult to obtain results for lower heat fluxes (below $T_{\lambda}$ ).

The HT measurement of the coil segment was an attempt to test a real coil in a smaller scale experiment with more freedom in power setting and in sampling. As performed, the experiment suffered some flaws but proved feasible and promising. Comparison with the CEA data indicates the possibility of heat leaks from the sample extremities. Once the technological problems are solved, the method could give new information about HT in $\mathrm{SC}$ coils.

\section{ACKNOWLEDGMENT}

The authors wish to thank A. Bastos Marzal, A. L. Dillenschneider, J. L. Servais, and P. F. Jacquot (CERN) for their help during preparation of the experiment. Field maps for the HRRQ analysis were prepared by B. Auchmann (CERN). C. de Montmollin kindly drew the Fig. 6. D. Richter had pleasure in learning about mechanical matters and magnet production from P. Fessia and H. Kummer, and in discussing magnet dynamics with A. P. Verweij (CERN).

Authors are grateful to L.-R. Oberli, L. Rossi, and P. Lebrun of CERN for their continuous support.

\section{REFERENCES}

[1] L. Rossi, "The Large Hadron Collider and the Role of Superconductivity in one of the Largest Scientific Enterprises," presented at this conference.

[2] R. Perin et al., "Status of the arge Hadron Collider magnet development," in Proceedings of MT13, Victoria, 1993.

[3] N. Andreev et al., "Present state of the single and twin aperture short model program for the LHC," in Proceedings of MT15, Beijing, 1997.

[4] B. Baudouy, "Etude des Transferts de Chaleur dans les Isolations électriques de Câbles Supraconducteurs d'aimant d'accélérateur Refroidi par hélium Superfluide," Thèse de doctorat de l'Université Paris VI, Paris, 1996.

[5] C. Meuris, "Heat transport in insulation of cables cooled by superfluid helium," Cryogenics, vol. 31, pp. 624-628, 1991.

[6] L. Burnod et al., in Workshop on LHC Technology, CERN, Chamonix, 1993.

[7] L. Burnod et al., "Thermal modeling of the LHC dipoles functioning in superfluid helium," in Proc EPAC'94, 1994, pp. 2295-2297 [Online]. Available: http://www.jacow.org/

[8] C. Meuris et al., "Heat transfer in electrical insulation of LHC cables cooled with superfluid helium," Cryogenics, vol. 39, pp. 921-931, 1999.

[9] A. P. Verweij, ElectroDynamics of Superconducting Cables in Accelerator Magnets PhD thesis, University of Twente, The Netherlands, 1995.

[10] T. Ogitsu, A. Devred, and V. Kovachev, "Influence of inter-strand coupling current on field quality of superconducting accelerator magnets," Particle Accelerators, vol. 57, pp. 215-235, 1997.

[11] D. Richter et al., "DC measurement of electrical contacts between strands in superconducting cables for the LHC main magnets," IEEE Trans. Appl. Supercond., vol. 7, no. 2, pp. 786-792, 1997.

[12] K. Artoos et al., "Status of the short dipole model program for the LHC," IEEE Trans. Appl. Supercond., vol. 10, no. 1, pp. 49-52, March 2000.

[13] G. Kirby et al., "Power test results of the first LHC second generation superconducting single aperture $1 \mathrm{~m}$ long dipole models," in Proc. ICEC 16, Kitakyushu, 1996.

[14] S. Sanfilippo et al., "Training quench performance and quench location of the short superconducting dipole models for the LHC," IEEE Trans. Appl. Supercond., vol. 12, no. 1, pp. 251-254, March 2000.

[15] L. Bottura, "A practical fit for the critical surface of NbTi," IEEE Trans. Appl. Supercond., vol. 10, no. 1, pp. 1054-1057, March 2000.

[16] Z. Ang et al., "Measurement of AC loss and magnetic field during ramps in the LHC model dipoles," IEEE Trans. Appl. Supercond., vol. 9, no. 2, pp. 742-745, June 1999.

[17] T. W. Kerlin, Practical Thermocouple Thermometry, North Carolina, ISA Publishing, 1999.

[18] T. Boutbul et al., "Critical current test facilities for LHC superconducting NbTi cable strands," in Proceedings of the 5th EUCAS, Copenhagen, 2001. 